\title{
Electronic Excitations in Liquid Helium: The Evolution from Small Clusters to Large Droplets
}

\author{
M. Joppien, ${ }^{1}$ R. Karnbach, ${ }^{1}$ and T. Möller ${ }^{2}$ \\ 'II. Institut für Experimentalphysik, Universität Hamburg, Luruper Chaussee 149, 22761 Hamburg, Federal Republic of Germany \\ ${ }^{2}$ Hamburger Synchrotronstrahlungslabor HASYLAB at Deutsches Elektronensynchrotron, \\ DESY (Hamburg), 22607 Hamburg, Notkestrasse 85, Federal Republic of Germany
}

(Received 30 April 1993)

\begin{abstract}
The absorption coefficient of liquid He droplets is reported in the energy range from the first electronic excitation up to the ionization limit. It is obtained from total fluorescence yield curves. The size of $\mathrm{He}$ droplets is varied between approximately $10^{6}$ atoms/particle to small clusters containing on the order of 50 atoms. The character of the excited states is discussed in view of different theoretical approaches, i.e., molecular excitation, Frenkel and Wannier type excitons. The Frenkel and Wannier model, which is very successful for the description of excited states in insulators, has severe shortcomings in the case of liquid He. Furthermore, the implications for the structural peculiarities of liquid $\mathrm{He}$ are addressed.
\end{abstract}

PACS numbers: 78.20.Dj, 36.40.+d, 67.90. $+\mathrm{z}, 71.90 .+\mathrm{q}$

Among the elements of the periodic table $\mathrm{He}$ has an exceptional position. It is a very interesting subject for experimental and theoretical investigations in the gas phase as well as in the condensed phase [1]. In view of the electronic structure $\mathrm{He}$ is one of the simplest atoms; it is therefore ideally suited for model calculations because of the small number of electrons. The closing of the $1 s$ shell is responsible for the large ionization potential which is the highest of all atoms. The condensed phase is particularly interesting because $\mathrm{He}$ remains liquid even at $0 \mathrm{~K}$.

As a result of these peculiarities, our knowledge of the optical properties and the electronic excitations in condensed $\mathrm{He}$ is rather poor. Up to now the absorption spectrum of liquid or solid He has not been measured. This is because no transparent window materials are available in the spectral range of interest which can withstand the high vapor pressure. Even the reflectivity is known only in small segments energetically close to a few atomic resonance lines [2].

He clusters prepared in a supersonic beam allow a detailed investigation of the optical properties and electronic excitations of this interesting fluid. Furthermore, He clusters themselves are very interesting subjects from experimental [3-5] and theorotical [6-8] points of view.

So far, most of the experimental and theoretical investigations focus only on the geometrical structure and the thermodynamic properties of He clusters. However, since He is one of the simplest atoms, it is a challenge to explore the evolution of excited energy levels when the size is varied between the atomic and the bulk limit. For the heavier rare gases similar investigations were recently performed and turned out to be very fruitful for the understanding of how electronically excited energy levels evolve with size $[9,10]$.

In this Letter we present fluorescence excitation spectra of He clusters containing information which is almost identical with the absorption. To get a better understanding of the nature of the electronically excited states their evolution is investigated as a function of cluster size.
Excitation with photons offers the possibility to study directly the properties of neutral He clusters. The character of the excited states differs remarkably from that of electronic excitation in clusters and solids of the heavier rare gases.

The measurements were performed at the experimental station Clulu at HASYLAB (Hamburg) [11]. The experimental method is essentially the same as in previous studies on the heavier rare gas clusters [9]. The nozzle, however, is now mounted on a liquid He cryostat. Helium clusters are prepared in a nozzle expansion starting either from the liquid or the gas phase (nozzle diameter 20-100 $\mu \mathrm{m}$, stagnation pressure up to $100 \mathrm{bars}$, temperature $T=4-15 \mathrm{~K}$ ) and are photoexcited with tunable synchrotron radiation (bandpass $0.1 \mathrm{~nm}$ ). The estimated temperature of the clusters is approximately $0.4 \mathrm{~K} \mathrm{[3].}$ Fluorescence light is detected with a closed channel plate detector (aluminum foil window, $40 \mathrm{~nm}$ thick) which is sensitive between 15 and $30 \mathrm{eV}$. In the case of He clusters a precise determination of the size is very difficult because of their fragility. Here we give only rough estimates of the size based on results for the heavier rare gas clusters [11] and well known scaling laws [12].

Before we present our results we would like to give a brief overview of the different types of electronic excitations in dense van der Waals bound materials according to Ref. [13]. Electronic excitations in dense gases are directly related to the corresponding excitations in the isolated atoms or molecules. While the positively charged core (hole) is nearly unaffected, the excited electron is perturbed by the neighboring atoms. This leads to a broadening of the absorption lines. If the particle density exceeds a critical value, the character of the excitation changes [13] fundamentally. The positively charged hole is no longer located at one atom. This is a signature of the exciton concept. Depending on the ratio between the radius of excitonic orbitals and the nearest-neighbor distance, two limiting cases are important. The Frenkel case is characterized by small size tightly bound states. In the Wannier model the conduction band electrons and 
valence band holes form bound electron hole states which are usually treated in the effective mass approximation. They form a series of excited states similar to that of the hydrogen atom characterized by a main quantum number $n$ starting with $n=1$. The Wannier model is only appropriate if the radius of the orbitals is large compared to the nearest-neighbor distance. Strictly speaking, the exciton description is only valid for solids with translational symmetry [13]. However, excitons described in the Frenkel as well as in the Wannier model are observed in very dense gas and liquid rare gases [13].

Figure 1 shows fluorescence excitation spectra of $\mathrm{He}$ droplets (a) and large clusters (b) (estimated size $N$ $\sim 10^{4}$ atoms/cluster) in comparison with a fluorescence excitation spectrum of dense He gas (c) [14]. Below the ionization limit $(\sim 24.6 \mathrm{eV}$ for large clusters [4]) these spectra are expected to correspond to the absorption coefficient because dark relaxation channels are expected to be of minor importance. For large droplets (from the fragmentation of the liquid [4]), however, saturation

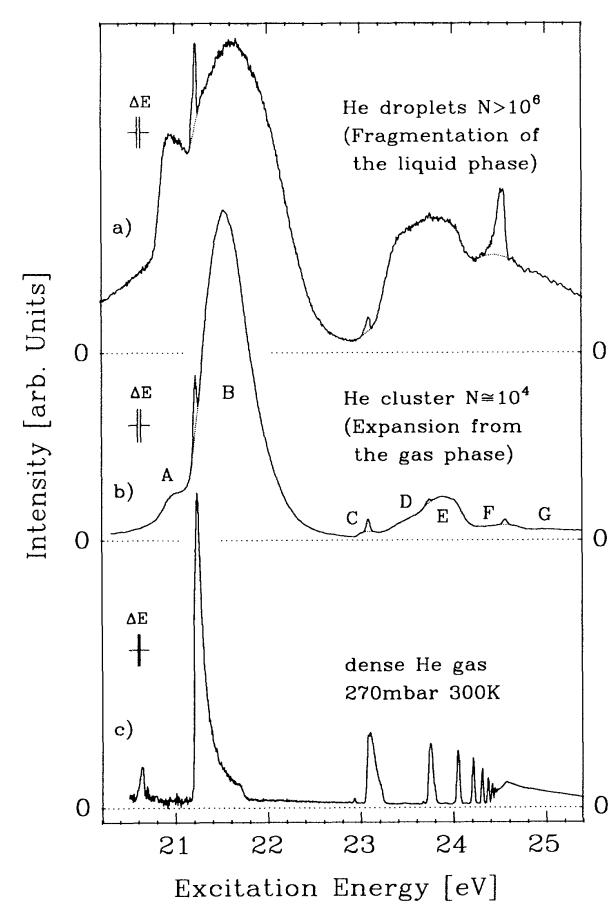

FIG. 1. Fluorescence excitation spectra of (a) large He droplets $(20 \mu \mathrm{m}$ nozzle, 7.5 bars, $T=5 \mathrm{~K}$ expanded from the liquid phase), (b) large He clusters ( $80 \mu \mathrm{m}$ nozzle, 2.5 bars, $T=8 \mathrm{~K}$, expansion from the gas phase), and (c) dense He gas [14] enclosed in a gas cell $(270$ mbar, $300 \mathrm{~K})$. The sharp lines in (a) and (b) are due to atomic transitions $1 s \rightarrow n p$. The same is true for the sharp line at $-24.56 \mathrm{eV}$ which is a congestion of many lines close to the ionization limit falling into the bandpass of excitation. It is rather intense because the background gas is at the position of optically thick atomic resonance lines. The dotted line is only to guide the eye. For the labeling of the bands see text. effects and resonant scattering distortions have to be considered. Both effects are clearly visible in the spectrum in Fig. 1(a). As a consequence of the saturation effects, the dynamic range in Fig. 1(a) is much smaller than in Fig. 1(b). Furthermore, the large signal energetically below $20.8 \mathrm{eV}$ is due to light scattering at the large droplets. This has been proved by time resolved measurements. Based on a comparison with measurements of scattering of light from $\mathrm{Kr}$ clusters [15], the estimated cluster size is in the order of $10^{6}$ atoms/cluster or even larger in accordance with Ref. [4]. Apart from the deviations mentioned above, the gross spectral features in Figs. 1(a) and 1(b) are very similar. We therefore conclude that the spectrum in Fig. 1(b) which is free of these distortions should show a close correspondence to the absorption coefficient of liquid He.

The spectrum in Fig. 1(c) recorded on dense gas can be well interpreted in terms of a molecular description. Only one series of transitions is observed starting from the $1 s$ orbital into molecular states with atomic $n p$ states as the dissociation limit. Pronounced molecular wings on the high energy side of the position of the atomic resonance lines are due to free-free transitions into excited states with a "hump" in the potential curve [14].

The absorption of liquid He looks very different. The spectrum of large clusters [Fig. 1(b)] shows several very broad bands (FWHM up to $0.55 \mathrm{eV}$ ). These bands are labeled by capital letters. A few sharp, weak lines visible in the spectrum are due to atomic $\mathrm{He}$ which is always present in the beam. In the following we try to give a detailed assignment of the absorption bands. In analogy to absorption spectra of other liquid and solid rare gases [13], one might be tempted to interpret the observed bands in an exciton description. It will be shown below that for He the exciton description has severe shortcomings. He is markedly different from the other rare gases and new theoretical approaches are needed.

The strong absorption bands $A$ and $B$ between 20.6 and $22.9 \mathrm{eV}$ are assigned to molecular transitions into excited states related to atomic $2 s$ and $2 p$ states. Similar spectral features are also observed in the reflectivity curves of liquid $\mathrm{He}$ [2]. In the molecular description band $B$ corresponds to the first strong molecular absorption band in the dense gas [Fig. 1(c)]. The large spectral shift $(\sim 0.43 \mathrm{eV})$ relative to the atomic resonance transition $(1 s \rightarrow 2 p)$ reflects the repulsive interaction between the excited electron and the neighboring $\mathrm{He}$ atoms. For this excitation the Frenkel exciton model seems to be inappropriate because the density of liquid $\mathrm{He}$ is very small. It should be noted that no substructure similar to that of excitons in the other rare gases (splitting into bulk and surface states, longitudinal-transverse splitting of the bulk excitons [13]) is observed. The large width (FWHM $=0.55 \mathrm{eV}$ ) of the band and the absence of fine structure gives strong evidence for large density fluctuations in liquid $\mathrm{He}$. Furthermore, it indicates that $\mathrm{He}$ clusters do not have a well defined sharp surface. 
Band $A$ is presumably related to the dipole forbidden $1 s \rightarrow 2 s$ atomic transition which gains oscillator strength in the molecule for small internuclear separations. In the spectrum recorded in the gas phase it is clearly visible at $20.6 \mathrm{eV}$. In the energy range $23-25 \mathrm{eV}$ absorption bands are observed which have no clear atomic or molecular parentage. They will be discussed in the context of the evolution of electronic excitation with cluster size.

Fluorescence excitation spectra of helium clusters recorded as a function of size are presented in Fig. 2. The cluster size estimated from the scaling laws [12] ranges between 50 and 5000 atoms/cluster. A comparison with similar measurements [16] recently performed where the size of the cluster is determined directly in a scattering experiment [17] shows that these numbers are of the correct order of magnitude.

For small clusters [Figs. 2(a) and 2(b)] each of the atomic resonance lines is followed by a wing on the high energy side. These spectra are similar to the spectrum recorded in the dense gas [Fig. 1(c)] where the bands are assigned to molecular free-free transitions [14]. With in-

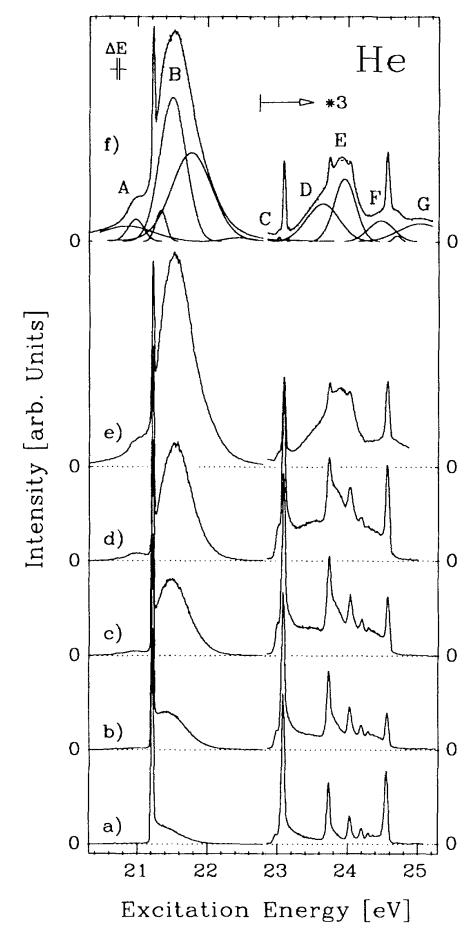

FIG. 2. Fluorescence excitation spectra of He cluster produced in an adiabatic expansion from the gas phase through an $80 \mu \mathrm{m}$ flat nozzle. Expansion conditions and estimated average cluster size: (a) $p=375 \mathrm{mbar}, T=6 \mathrm{~K} ; N=50$; (b) $450 \mathrm{mbar}$, $6 \mathrm{~K}$; 120 ; (c) 550 mbar, $6 \mathrm{~K}$; 200; (d) $1150 \mathrm{mbar}, 7.6 \mathrm{~K}$; 500; (e) 1500 mbar, $7.5 \mathrm{~K}$; 1750; (f) 2.25 bars, $8 \mathrm{~K}$; 5000. The sharp lines are due to transitions $1 s \rightarrow n p$ of $\mathrm{He}$ atoms being always present in the beam (see Fig. 1). For the labeling of the band and the discussion of the fits see text. For clarity atomic contributions used in the fitting procedure are not shown in ( $f$ ). creasing cluster size the absorption bands of the clusters change considerably. To get more quantitative results the measured spectra are fitted with asymmetric Gaussians and Lorentzians. For the large clusters $(N \sim 5000)$ the fits are included in Fig. 2(f). We start with the discussion of the bands related to the atomic $1 s \rightarrow 2 p$ transition. The shape of the wing on the high energy side changes from a highly asymmetric wing to a more symmetric band peaked at $21.5 \mathrm{eV}$. Even for the large clusters [Fig. 2(f)] high quality fits can only be obtained with three or more Gaussians. The band $A$ at $\sim 21 \mathrm{eV}$ related to the $1 s \rightarrow 2 s$ transition which becomes allowed at short internuclear separations appears only for large clusters $(N>100)$. This indicates that the average internuclear distance in $\mathrm{He}$ clusters decreases with increasing cluster size in agreement with theoretical work [6-8].

The assignment and the interpretation of absorption bands in the range $23-25 \mathrm{eV}$ is rather complicated for three reasons: (1) There is strong overlap of the bands. (2) The shape of the bands is unusual and changes with size. (3) The number of bands changes with size. In the following we focus on bands $D$ and $E$ which are the dominant absorption bands. For large clusters we have fitted bands $D$ and $E$, respectively, with only one asymmetric Gaussian. It is obvious that such a procedure is inappropriate for small clusters. Small clusters show molecular wings on the high energy side of each atomic resonance line. The width of these wings increases with increasing cluster size. Medium sized clusters $[N=500$, Fig. 2(d)] show pronounced absorption bands ( $D$ and $E$ ) similar to band $B$ with maxima at 23.45 and $23.85 \mathrm{eV}$, respectively. For the cluster size under discussion $(N=500)$ they seem to be related to the atomic $3 p$ and $4 p$ states, respectively. However, if the cluster size is increased further the intensity of band $E$ increases compared to band $D$ (1.2 times more intense than band $D$ for $N=5000$ ). This makes an interpretation in a molecular or Frenkel exciton description unfavorable because the strength of electronic transitions usually decreases with a high power of the main quantum number $n$ [13].

For the largest clusters [Figs. 1(b) and 2(f)] the absorption bands in the energy range $23-25 \mathrm{eV}$ do not have a clear atomic parentage. In analogy to the heavier rare gases $[10,13]$, one might be tempted to assign these weakly bound excitations close to the ionization limit to Wannier excitons. However, in the case of liquid $\mathrm{He}$ a clear exciton series being the signature of Wannier excitons is not visible. In the Wannier model the states are characterized by their main quantum number $n$, starting with $n=1$ in analogy to the hydrogen atom. In this description the strong absorption band $E$ should correspond to the $n=2$ exciton (for the energetically lowest $n=1$ exciton state the Wannier description also fails for the heavier rare gases [13]). With reasonable assumptions for the effective electron and hole mass (see Table I) and a band gap energy of $25.5 \mathrm{eV}$ [4] the calculated transition energies for the $n=2$ state ranges between 22.4 and 23.0 
TABLE I. Wannier exciton radii $r_{w}=a_{0} \varepsilon n^{2} / \mu$ and binding energies $B_{n}=B_{0} \mu / \varepsilon^{2} n^{2}$ calculated in the Wannier model [13] in comparison with the radius of orbitals of $\mathrm{He}$ atoms $r_{a}$ (quantum defect $\delta=0$ for atomic $n p$ states) and experimentally determined binding energies $B_{\exp }(\varepsilon$ and $\mu$ are the dielectric constant and the reduced exciton mass and $a_{0}$ and $B_{0}$ are the Bohrs radius and the hydrogen binding energy, respectively). For the determination of the binding energy a band gap energy of 25.5 $\mathrm{eV}$ [4] is assumed. For the lowest electronically excited state $n_{w}=1$ corresponds to $n_{a}=2$. Parameters: dielectric constant $\varepsilon=1.035$ [4]; since information on the effective electron and hole masses is not available two different sets of values are taken for the reduced exciton mass (a) $\mu=m_{e}$ where $m_{e}$ is the free electron mass (heavy hole approximation) and (b) $\mu=0.8 m_{e}$ which is the value for solid $\mathrm{Ne}$ [13].

\begin{tabular}{|c|c|c|c|c|c|c|c|}
\hline \multirow[t]{2}{*}{$n_{w}$} & \multicolumn{2}{|c|}{$r_{w}(\AA)$} & \multicolumn{2}{|c|}{$B_{n}(\mathrm{eV})$} & \multirow[t]{2}{*}{$n_{a}$} & \multirow[t]{2}{*}{$r_{a}(\AA)$} & \multirow{2}{*}{$\begin{array}{l}B_{\exp }(\mathrm{eV}) \\
\text { This work }\end{array}$} \\
\hline & (a) & (b) & (a) & (b) & & & \\
\hline 1 & 0.548 & 0.684 & 12.7 & 10.26 & 2 & 2.11 & $3.85($ Band $B)$ \\
\hline 2 & 2.19 & 2.74 & 3.14 & 2.54 & 3 & 4.76 & $1.55($ Band $E)$ \\
\hline 3 & 4.93 & 6.15 & 1.41 & 1.13 & 4 & 8.46 & \\
\hline
\end{tabular}

$\mathrm{eV}$ which is far below the observed value of $23.95 \mathrm{eV}$ for band $E$. This discrepancy becomes more clear when the calculated binding energies of $2.54-3.17 \mathrm{eV}$ are contrasted with the experimental binding energy of $1.55 \mathrm{eV}$ of band $E$.

The failure of the Wannier description even for the $n=2$ state is not surprising because the exciton radius $r_{w}$ calculated for the first two members in the Wannier model is smaller than the nearest-neighbor distance of the liquid of $\sim 3.5 \AA$ [1]. Calculated values are summarized in Table I. Even for the third state (main quantum number $n=3$ ) the calculated exciton radius is close to the nearest-neighbor distance in liquid He. This is a direct consequence of the small density of liquid $\mathrm{He}$ and of the small dielectric constant. On the other hand, the radii of excited $\mathrm{He}$ atoms in the $3 p$ and $4 p$ states, respectively, are close to the nearest-neighbor distance. Consequently, a molecular or the Frenkel exciton description where the $1 s$ hole and excited electron [13] are located at the same atom is also inappropriate.

Our results show that the electronic properties of liquid He differ remarkably from that of the heavier rare gases $[9,10,13]$ in the sense that other theoretical concepts have to be applied. In particular, the Wannier and Frenkel exciton model which successfully describes electronic excitations in rare gas solids fails in the case of liquid He. On the other hand, also molecular concepts where the atomic parentage of the states is still visible have severe limitations for states with high main quantum numbers. Finally, we would like to mention that pronounced surface states similar to those in the heavier rare gases
$[10,13]$ are not observed. This is a strong indication that He clusters do not have a well defined sharp surface. Results presented here for the excitation processes in liquid He are only a first step towards the understanding of the electronic properties of this highly interesting fluid. However, they already reflect several unusual properties which might stimulate theoretical work.

We would like to thank J. Gspann (Karlsruhe) and the group of J. P. Toennies (Göttingen) for helpful discussions concerning the preparation of He clusters. Furthermore, we are grateful to R. Fröchtenicht (Göttingen) for making available experimental data prior to publication. Financial support of the Bundesministerium für Forschung und Technologie under Grant No. 055 GUAY1 TP1 is kindly acknowledged.

[1] J. Wilks, Liquid and Solid Helium (Clarendon, Oxford, 1967).

[2] C. M. Surko, G. J. Dick, F. Reif, and W. C. Walker, Phys. Rev. Lett. 23, 842 (1969).

[3] J. Gspann, in Physics of Electronic and Atomic Process$e s$, edited by S. Datz (North-Holland, Amsterdam, 1982), p. 79ff.

[4] H. Buchenau, J. P. Toennies, and J. A. Northby, J. Chem. Phys. 95, 8134 (1991).

[5] S. Goyal, D. L. Schutt, and G. Scoles, Phys. Rev. Lett. 69, 933 (1992).

[6] S. Stringari and J. Treiner, J. Chem. Phys. 87, 5021 (1987).

[7] C. L. Cleveland, U. Landmann, and R. N. Barnett, Phys. Rev. B 39, 117 (1989).

[8] M. V. Rama Krishna and K. B. Whaley, J. Chem. Phys. 93, 6738 (1990), and references therein.

[9] T. Möller, Z. Phys. D 20, 1 (1991).

[10] J. Wörmer, M. Joppien, G. Zimmerer, and T. Möller, Phys. Rev. Lett. 67, 2053 (1991).

[11] R. Karnbach, M. Joppien, J. Stapelfeldt, J. Wörmer, and T. Möller (to be published).

[12] O. F. Hagena and W. Obert, J. Chem. Phys. 56, 1793 (1972).

[13] N. Schwentner, E. E. Koch, and J. Jortner, Electronic Excitations in Condensed Rare Gases (Springer, Berlin, 1985).

[14] V. Guzielski, M. C. Castex, J. Wörmer, and T. Möller, Chem. Phys. Lett. 179, 243 (1991); the spectrum taken from this work has been corrected for the transmission of the window and saturation effects.

[15] J. Wörmer, M. Joppien, and T. Möller, Chem. Phys. Lett. 182, 632 (1991).

[16] R. Fröchtenicht, A. Ding, T. Drewello, M. FieberErdmann, U. Henne, and J. P. Toennies (to be published).

[17] M. Lewerenz, B. Schilling, and J. P. Toennies, Chem. Phys. Lett. 206, 381 (1993). 